\title{
Susceptibility of Streptococcus pyogenes to azithromycin, clarithromycin, erythromycin and roxithromycin in vitro
}

\author{
G. J. VAN ASSELT*, J. H. SLOOS, R. P. MOUTON, C. P. A. VAN BOVEN \\ and J. A. M. VAN DE KLUNDERT
}

Department of Medical Microbiology, University Hospital Leiden, PO Box 9600, 2300 RC Leiden, The Netherlands

\begin{abstract}
Summary. The susceptibility of 180 clinical isolates of Streptococcus pyogenes from six regions of The Netherlands to the macrolide antibiotics azithromycin, clarithromycin, erythromycin and roxithromycin was analysed. The results of a microbroth MIC method, the E-test method and a disk diffusion assay were compared, and the MBC determined. In addition, the susceptibility to erythromycin of 436 clinical isolates of $S$. pyogenes from the Leiden region was determined. The microbroth MIC90s of azithromycin, clarithromycin, erythromycin and roxithromycin for group A streptococci were $\leqslant 0.5 \mathrm{mg} / \mathrm{L}$. Erythromycin had the lowest MIC $90(0.09 \mathrm{mg} / \mathrm{L})$. The MIC data obtained with the E-test method suggested that clarithromycin and erythromycin had slightly higher anti-streptococcal activity than azithromycin and roxithromycin in vitro. MICs obtained with the E-test were lower than those found with the microbroth method. Only minor discrepancies were observed among the three methods. The MBC50 for both clarithromycin and erythromycin was $0.75 \mathrm{mg} / \mathrm{L}$ and $5.0 \mathrm{mg} / \mathrm{L}$ for azithromycin and roxithromycin. None of the 180 strains and two of the collection of 436 strains $(0.5 \%)$ were resistant to erythromycin and the other macrolides tested; MICs ranged from 1 to $16 \mathrm{mg} / \mathrm{L}$. The erythromycin-resistant strains showed an inducible type of macrolide-lincosamide-streptogramin B (MLS) resistance.
\end{abstract}

\section{Introduction}

The incidence of severe Streptococcus pyogenes infections including rheumatic fever has increased in the last decade $;{ }^{1.2}$ Some of these group A streptococcal infections can have a fatal outcome due to the production of pyrogenic exotoxin. ${ }^{2}$ However, most group A streptococcal infections appear to be no more severe than in previous years. ${ }^{3}$ Because persistence of $S$. pyogenes in the throat can lead to the development of rheumatic fever in some cases, eradication of these bacteria after pharyngotonsillitis is considered to be important. $^{4-6}$

Penicillin is still the drug of choice for treatment of infections with group A streptococci, but despite the uniform sensitivity to penicillin of group A streptococci, failures in the penicillin treatment of pharyngotonsillitis may occur. ${ }^{7}$ This can be due to the phenomenon of penicillin tolerance in the streptococci ${ }^{8.9}$ or to $\beta$-lactamase production by the sur-

Received 22 April 1995: accepted 25 April 1995.

*Present address and address for correspondence: Department of Medical Microbiology, Westeinde Hospital, Lijnbaan 32. 2512 VA Den Haag, The Netherlands. rounding flora. ${ }^{10.11}$ Erythromycin, a bacteristatic drug, is the alternative treatment for group A streptococcal infections if penicillin fails. It is also useful for infections caused by many gram-positive organisms in patients allergic to penicillin.

Erythromycin, a well established, safe and effective antimicrobial agent, has been the subject of renewed interest over the last decade because of its activity against respiratory tract pathogens, such as Legionella pneumophila, Mycoplasma pneumoniae and Chlamydia spp. New macrolide antibiotics are now available with an expanded spectrum and better pharmacological properties than erythromycin. ${ }^{12-14}$ Mainly because of the improved gastrointestinal tolerance of azithromycin, clarithromycin and roxithromycin, compared to erythromycin, these macrolides could be used as alternatives to penicillin. Some of these new macrolides are becoming the antibiotics of first choice in some clinics for treatment of respiratory tract, ear and throat infections, because of their activity against Haemophilus influenzae. ${ }^{11}$ Because of this increased use in empirical therapy, the effect of various macrolides in group A streptococcal infections is of interest. Few reports exist on this. ${ }^{15}$

Resistance of group A streptococci to erythromycin 
has been found in several countries, and rates range from 0.5 to $60 \%$. The highest rates were in $\mathrm{Japan}^{16}$ and certain areas in Finland. ${ }^{17}$ Data from The Netherlands has not been published yet.

The purpose of the present study on group A streptococci was: to determine both the minimal inhibitory concentration (MIC) and the minimal bactericidal concentration (MBC) of erythromycin, azithromycin, clarithromycin and roxithromycin for group A streptococci; to compare the results of the traditional microbroth MIC determination and the recently available E-test and to correlate both with the outcome of the disk diffusion assay; and to estimate the rate of erythromycin resistance in The Netherlands.

\section{Materials and methods}

\section{Strains}

Two separate collections of clinical isolates were examined: one collection comprised 180 group A streptococci from throat and pus cultures performed in hospital laboratories in six areas of The Netherlands (30 strains each from Leiden, Den Haag, Rotterdam, Utrecht, Deventer and Enschede). These unrelated clinical isolates were collected consecutively during a period of several months in 1993. The second collection comprised 436 clinical isolates of group A streptococci, obtained from different clinical specimens in the period 1988-1990 in the Leiden region.

$\beta$-Haemolytic colonies were identified as group A streptococci by a latex agglutination test (Streptex; Wellcome Diagnostics, Dartford). The strains were maintained in glycerol broth at $-70^{\circ} \mathrm{C}$ until all isolates were collected. Strains from one laboratory were stored after lyophilisation. Stocks of group A streptococci were subcultured twice on to defibrinated sheep blood $5 \%$ agar (Oxoid and BioTrading) before susceptibility testing. Enterococcus faecalis strain ATCC $^{\mathrm{T}} 29212$ (erythromycin MIC $2 \mathrm{mg} / \mathrm{L}$ ) and $S$. pyogenes strain Otto (\# 350; erythromycin MIC > $64 \mathrm{mg} / \mathrm{L}$ ), obtained from H. Seppälä (Finland), were included as controls.

\section{Antibiotics}

Antibiotic preparations were obtained from their respective manufacturers: azithromycin from Pfizer Inc., New York, USA; clarithromycin and erythromycin from Abbott Laboratories, Abbott Park, IL, USA; roxithromycin from Roussel Uclaf, Paris, France. E-test strips of azithromycin, clarithromycin, erythromycin and roxithromycin were obtained from AB Biodisk, Solna, Sweden. Disks of azithromycin, clarithromycin and erythromycin were supplied by BBL (BBL Sensi-Disc, Becton Dickinson Microbiology Systems, Cockeysville, MD, USA); disks of roxithromycin from Oxoid (Unipath Limited, Basingstoke, Hampshire). Disks contained $15 \mu \mathrm{g}$ of antibiotic. Disks of penicillin $(10 \mu \mathrm{g})$ were obtained from $\mathrm{BBL}$ and tetracycline $(30 \mu \mathrm{g})$ and clindamycin $(10 \mu \mathrm{g})$ from Oxoid.

\section{$M I C$ and $M B C$ determinations}

The MICs of azithromycin, clarithromycin, erythromycin and roxithromycin for the 180 isolates were determined by a broth microdilution method. Serial two-fold dilutions of antimicrobial drug solutions were added to Mueller-Hinton broth ( $\mathrm{pH} 7 \cdot 3)$ to achieve concentrations, after addition of the cells, from $0.0039 \mathrm{mg} / \mathrm{L}$ to $4.0 \mathrm{mg} / \mathrm{L}$ for all macrolides. For resistant strains the range was extended to $128 \mathrm{mg} / \mathrm{L}$. A volume of $0.05 \mathrm{ml} /$ well of each antimicrobial agent was introduced into the wells of the microdilution plates. Plates were stored at $-20^{\circ} \mathrm{C}$ until used. The plates were inoculated by adding $0.05 \mathrm{ml}$ to yield a final inoculum of $10^{5} \mathrm{cfu} /$ well $\left(10^{6} \mathrm{cfu} / \mathrm{ml}\right)$. The plates were covered and incubated in air at $37^{\circ} \mathrm{C}$ for $18-20 \mathrm{~h}$; the MICs, defined as the lowest concentration of each antibiotic that completely inhibited visible growth, were read visually after the incubation period.

To determine the MBC, $1 \mu \mathrm{l}$ of the MIC dilutions from each well was applied with a multipoint inoculator to the surface of agar plates containing sheep blood $5 \% \mathrm{v} / \mathrm{v}$. The plates were incubated for $24 \mathrm{~h}$ in air at $37^{\circ} \mathrm{C}$. The $\mathrm{MBC}$ was defined as the lowest antibiotic concentration that did not yield visible colonies, i.e., killed $>99.9 \%$ of the initial inoculum. All tests were repeated once on a separate occasion. Both values were taken into account.

The MIC susceptibility breakpoints were $1 \mathrm{mg} / \mathrm{L}$ for erythromycin, ${ }^{18} 2 \mathrm{mg} / \mathrm{L}$ for azithromycin and roxithromycin, ${ }^{17,19}$ and $1 \mathrm{mg} / \mathrm{L}$ for clarithromycin. ${ }^{20}$

\section{E-test method}

The collection of 180 isolates was evaluated by means of the E-test method, according to the instructions of the manufacturer. The E-test yields a quantitative MIC determination by means of a strip containing an antibiotic gradient. The performance of the E-test is comparable to a disk diffusion assay. Etest strips were placed on sheep blood agar plates that had been inoculated with a cotton swab dipped into a bacterial suspension with a turbidity equivalent to that of a $0.5 \mathrm{McF}$ arland standard. Plates were incubated in air at $37^{\circ} \mathrm{C}$ for $18-24 \mathrm{~h}$. The MIC was read at the point of intersection of the zone edge with the E-test strip. All tests were repeated once on a separate occasion. Both values were taken into account.

\section{Agar disk diffusion assay}

The disk diffusion method was performed on blood agar base supplemented with sheep blood $5 \%$, with both Oxoid and BBL disks. For 180 strains, the disks of azithromycin, clarithromycin, erythromycin and roxithromycin were placed on plates inoculated to achieve semi-confluent growth. This was obtained by applying a 10-fold dilution of a bacterial suspension, equivalent to a turbidity of $0.5 \mathrm{McF}$ arland standard, 
to the plates with a cotton swab. After incubation for $18-24 \mathrm{~h}$ in air at $37^{\circ} \mathrm{C}$, the diameters of the inhibition zones were measured. All tests were repeated once on a separate occasion. Both values were taken into account.

The collection of 436 strains was tested for susceptibility to penicillin, erythromycin and tetracycline by the disk diffusion method as described above. For strains with an erythromycin zone diameter $<27 \mathrm{~mm}$, MICs were determined by the broth microdilution method, as described above, to distinguish between resistant and moderately susceptible strains. Strains were considered resistant when the inhibition zone was $<18 \mathrm{~mm}^{21}$

\section{Disk induction test}

Erythromycin-resistant strains were subjected to a disk induction test with erythromycin as inducer of clindamycin resistance. In this test, the distance between disks was $20-30 \mathrm{~mm}$. After incubation for $18-24 \mathrm{~h}$ at $37^{\circ} \mathrm{C}$, blurring of the zone around the clindamycin disk was considered to be evidence of inducible resistance. Resistance to erythromycin without blurring of the clindamycin zone of inhibition indicated constitutive resistance.

\section{Results}

The in-vitro activities (MICs) of the four macrolides against 180 group A streptococci assessed by the microbroth and E-test methods are summarised in the table. All strains were susceptible to erythromycin. In the disk diffusion assay, the mean of the zone diameters for azithromycin was $27 \mathrm{~mm}$ (range 23-42), for clarithromycin $31 \mathrm{~mm}$ (27-46), for erythromycin $31 \mathrm{~mm}$ $(27-43)$ and for roxithromycin $28 \mathrm{~mm}(24-42)$. The data for the three methods indicated that either erythromycin or clarithromycin was the most active. In view of its MBC50, the bactericidal activity of clarithromycin and to a lesser extent erythromycin was reached at a lower concentration than that of azithromycin and roxithromycin (table). The MICs of the macrolides obtained by the E-test were lower than those obtained by the microbroth method. Most of the MIC values obtained by the microbroth and E-test methods ranged within three dilution steps. The MICs of the great majority of strains were within narrow limits, as were those produced by the disk diffusion

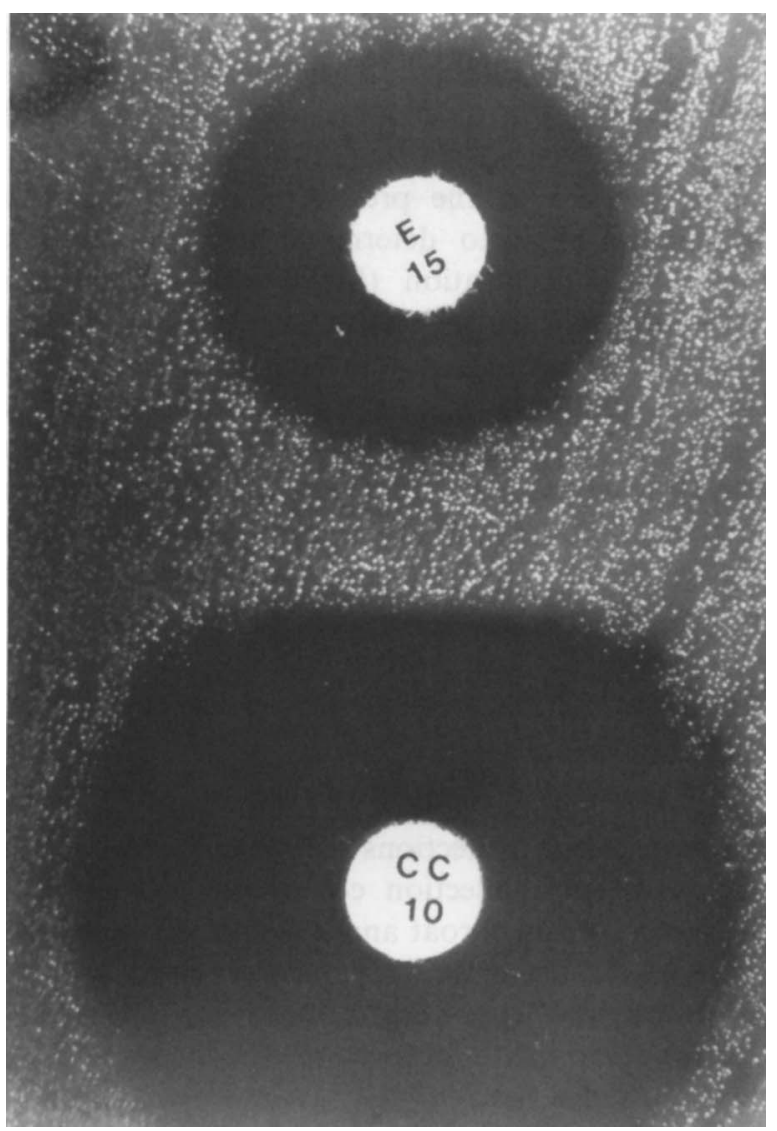

Figure. Disk agar susceptibility test (disk approximation) of a group A streptococcus with inducible MLS resistance. $\mathrm{CC}$, clindamycin $(10 \mu \mathrm{g}) ; \mathrm{E}$, erythromycin $(15 \mu \mathrm{g})$

assay. Because of this narrow range, regression analysis was not considered useful. Susceptibility tests were reproducible: for $\geqslant 95 \%$ of strains the outcome of the repeated experiments was within two dilution steps, except for the microbroth MIC tests for clarithromycin. The values of the repeated MBC experiments differed by more than two dilution steps for $27 \%$ of strains. The discrepant MIC and MBC determinations were excluded from the analysis.

The mean MIC of erythromycin against $E$. faecalis ATCC $^{\mathrm{T}} 29212$ was $2.57 \mathrm{mg} / \mathrm{L}$ (range 2.0-3.0). These data corroborated the MIC given by the ATCC. The mean MIC of azithromycin against the enterococcus was $8.00 \mathrm{mg} / \mathrm{L}$ (range $6.0-12.0$ ); for clarithromycin it was $3 \cdot 21 \mathrm{mg} / \mathrm{L}(0 \cdot 5-6 \cdot 0)$ and for roxithromycin, $17 \cdot 14 \mathrm{mg} / \mathrm{L}(8 \cdot 0-24 \cdot 0)$.

No major differences in activity were found when strains that originated from six regions in The Netherlands were compared (data not shown).

Table. MIC and MBC for 180 erythromycin-susceptible group A streptococci from The Netherlands

\begin{tabular}{|c|c|c|c|c|c|c|c|c|c|}
\hline \multirow{2}{*}{ Macrolide } & \multicolumn{3}{|c|}{ MIC (mg/L) by E-test } & \multicolumn{6}{|c|}{ MIC and $\mathrm{MBC}(\mathrm{mg} / \mathrm{L})$ by microbroth method } \\
\hline & range & MIC 50 & MIC90 & MIC range & MIC50 & MIC90 & MBC range & MBC 50 & МBC 90 \\
\hline Azithromycin & $0.024-0.500$ & $0 \cdot 158$ & $0 \cdot 250$ & $0.06-0.63$ & $0 \cdot 12$ & $0 \cdot 31$ & $0.09-\geqslant 8.0$ & $5 \cdot 00$ & $\geqslant 8 \cdot 0$ \\
\hline Clarithromycin & $0.016-0.079$ & 0.032 & 0.047 & $0.05-0.31$ & 0.09 & $0 \cdot 16$ & $0.05-\geqslant 8.0$ & 0.75 & $\geqslant 8.0$ \\
\hline Erythromycin & $0.016-0.125$ & 0.056 & 0.094 & $0.05-0.31$ & 0.06 & 0.09 & $0.03 \geqslant 8.0$ & 0.75 & $\geqslant 8 \cdot 0$ \\
\hline Roxithromycin & $0.032-0.345$ & 0.158 & 0.250 & $0.06-0.63$ & $0 \cdot 12$ & $0 \cdot 31$ & $0.09-\geqslant 8.0$ & $5 \cdot 00$ & $\geqslant 8.0$ \\
\hline
\end{tabular}


The 436 consecutive isolates of group A streptococci from the Leiden region were all penicillin susceptible. For erythromycin the MIC 90 was $0 \cdot 12 \mathrm{mg} / \mathrm{L}$. Two of these strains exhibited low-level resistance to erythromycin as well as the 14-membered macrolides, clarithromycin and roxithromycin, and the 15 -membered macrolide, azithromycin; the MIC ranged from 1 to $16 \mathrm{mg} / \mathrm{L}$. The control strain obtained from H. Seppälä yielded MICs and MBCs for azithromycin, clarithromycin, erythromycin and roxithromycin of $\geqslant 256 \mathrm{mg} / \mathrm{L}$.

Both low-level erythromycin-resistant strains were susceptible to clindamycin but not azithromycin. The disk approximation test revealed induction of clindamycin resistance by erythromycin; the zone of inhibition of clindamycin was reduced on the erythromycin side of the disk (figure).

The two erythromycin-resistant strains were also tetracycline resistant. The overall tetracycline resistance rate for the 436 strains was $22 \cdot 7 \%$. This was constant in each year of the study period.

\section{Discussion}

The in-vitro anti-streptococcal activities of azithromycin and roxithromycin were similar; a similar situation was demonstrated for the more active macrolides, clarithromycin and erythromycin. For many of the isolates, all macrolides tested did not exhibit bactericidal activity within the concentration range used $(\mathrm{MBC} 90 \geqslant 8.0 \mathrm{mg} / \mathrm{L})$. The bactericidal concentrations of clarithromycin and erythromycin (MBC50) were the lowest of all the macrolides tested. On the basis of their MIC and MBC clarithromycin and erythromycin were the most active macrolides against group A streptococci in these tests.

When the E-test method was compared with the microbroth MIC method no major discrepancies were found. The slightly lower MIC values obtained by the E-test method than the microbroth method could be explained by the use of different media with different $\mathrm{pH}$ conditions (sheep blood agar versus MuellerHinton broth). Both the disk test and the E-test method are applicable in a diagnostic laboratory for susceptibility screening of group A streptococci for the macrolides tested in this study.

The non-homogeneous or granular suspensions of some of the isolates may have played a role in the variations in MBCs. This, together with the small volume of inoculum $(1 \mu \mathrm{l})$ deposited by the multipoint inoculator, could have caused some of the differences and small variations in $\mathrm{MBC}$; in the definition of the $\mathrm{MBC}$ a single surviving cfu determines the MBC. Because of this sampling error it is recommended that the sample volume should be at least $10 \mu \mathrm{l}$.

No erythromycin-resistant group A streptococci were found among the 180 strains from 1993, whereas the level of resistance for strains from the Leiden region isolated between 1988 and 1990 was $0.5 \%$. This low rate of resistance is in agreement with the cumulative data recorded during 1990-1992 in seven regional laboratories in The Netherlands. ${ }^{22}$

The two erythromycin-resistant strains had an inducible, rather than a constitutive, type of macrolide-lincosamide-streptogramin B (MLS) resistance. They proved to be susceptible to clindamycin but not to the 15-membered macrolide, azithromycin. ${ }^{23,24}$ Furthermore, these strains showed induction of clindamycin resistance by erythromycin, as demonstrated by the disk induction test (figure). The occurrence of inducible MLS resistance is in accordance with reports by other European and North American groups. Constitutive MLS resistance was first demonstrated in Japan $^{16}$ and only recently in Finland. ${ }^{17}$ Constitutive MLS-resistant strains are generally resistant to all macrolides, as was the reference strain obtained from H. Seppälä. ${ }^{25,26}$ According to several authors ${ }^{23,27,28}$ resistance to erythromycin and tetracycline may be genetically linked, but in this study no association between tetracycline and erythromycin resistance was found; $27.7 \%$ of strains from the Leiden region were tetracycline-resistant. All but two of these isolates were susceptible to erythromycin, indicating that for these strains resistance to erythromycin and tetracycline is not linked. This is in agreement with other findings. ${ }^{17,29,30}$

In Japan, Australia and Finland, the high incidence of resistance to erythromycin is due to a high usage of antibiotics. The rate of resistance of group A streptococci to erythromycin tends to decrease with decreasing macrolide use. ${ }^{16,31}$ The use of macrolides in animal feed has also led to selection of resistant organisms capable of transfer of their genetic material to group A streptococci. ${ }^{32}$ The low prevalence of resistant strains in this study may have resulted from a more restricted use of the macrolides. The rate of erythromycin-resistant group A streptococci ranges from 0.5 to $8 \%$ in Europe and North America. ${ }^{29.33-35}$ In some areas of Australia, Finland and Japan, resistance rates of $17.6 \%, 54 \%$ and $60 \%$, respectively, have been found. ${ }^{16,30,31}$

All macrolides tested in this study had a sufficient bacteristatic activity against group A streptococci, although the MIC and MBC data were slightly in favour of clarithromycin and erythromycin. Expansion of the spectrum of the new macrolides offers an advantage over erythromycin. ${ }^{12,13}$ The pharmacokinetics of the new macrolides are characterised by improved oral bioavailability, increased tissue penetration and persistence, and longer clearance times compared with those of erythromycin. ${ }^{14,36}$ The frequency of adverse effects of erythromycin, such as dose-related epigastric distress, may be reduced with the new macrolides.

The relative preference for the new macrolides in therapy for group A streptococcal infections depends on their in-vitro activity, comparative pharmacokinetics and, most importantly, clinical studies. In pharyngitis studies, in which one of the new macrolides 
is compared with penicillin, clinical cure or improvement is often the same for the two groups. ${ }^{37,38}$ However, penicillin treatment may possibly result in higher rates of successful eradication of group A streptococci from the throat. ${ }^{39}$ Furthermore, there is as yet no convincing evidence that a course of a macrolide will prevent acute rheumatic fever. ${ }^{40}$ In view of the lack of bactericidal action of the macrolides and the development of resistance under conditions of unrestricted use of these drugs, penicillin is still the therapy of first-choice for group A streptococcal infections. The macrolides may be used for this in the case of penicillin allergy in patients and penicillin tolerance in the streptococci.

\section{References}

1. Kaufhold A, Podbielski A, Kühnemund $O$, Lütticken $R$ Infektionen durch Streptococcus pyogenes: Neuere aspekte zur diagnostik, epidemiologie, klinik und therapie. Immun Infekt 1992; 20: 192-199.

2. Kaplan EL, Johnson DR, Cleary PP. Group A streptococcal serotypes isolated from patients and sibling contacts during the resurgence of rheumatic fever in the United States in the mid-1980s. J Infect Dis 1989; 159: 101-103

3. Denny FW. Group A streptococcal infections-1993. Curr Probl Pediatr 1993; 23: 179-185.

4. Bisno AL. Group A streptococcal infections and acute rheumatic fever. $N$ Engl J Med 1991; 325: 783-793.

5. Peter G. Streptococcal pharyngitis : current therapy and criteria for evaluation and new agents. Clin Infect Dis 1992; 14 Suppl 2: S218-223.

6. Vukmir RB. Adult and pediatric pharyngitis : a review. $J$ Emerg Med 1992: 10: 607-616.

7. Roos K, Holm SE, Ekedahl C. Treatment failure in acute streptococcal tonsillitis in children over the age of 10 and in adults. Scand $J$ Infect Dis $1985 ; 17: 357-365$.

8. Tuomanen E. Durack DT, Tomasz A. Antibiotic tolerance among clinical isolates of bacteria. Antimicrob Agents Chemother 1986; 30: 521-527.

9. Dagan R, Ferne M. Association of penicillin-tolerant streptococci with epidemics of streptococcal pharyngitis in closed communities. Eur J Clin Microbiol Infect Dis 1989; 8: 629-631.

10. Brook 1. The role of $\beta$-lactamase-producing bacteria in the persistence of streptococcal tonsillar infection. Rev Infect Dis 1984: 6: 601-607.

11. Brook 1. Penicillin failure and copathogenicity in streptococca pharyngotonsillitis $J$ Fam Pract 1994; 38: 175-179.

12. Kirst HA, Sides GD. New directions for macrolide antibiotics: structural modifications and in vitro activity. Antimicrob Agents Chemother 1989; 33: 1413-1418.

13. Bahal N, Nahata MC. The new macrolide antibiotics: azithromycin, clarithromycin. dirithromycin, and roxithromycin. Ann Pharmacouther 1992; 26: 46-55.

14. Rodvold KA, Piscitelli SC. New oral macrolide and fluoroquinolone antibiotics: An overview of pharmacokinetics, interactions, and safety. Clin Infect Dis 1993; 17 Suppl 1: S192-199.

15. Hamilton-Miller JMT. In-vitro activities of 14-, 15- and 16membered macrolides against Gram-positive cocci. $J$ Antimicrob Chemother 1992; 29: 141-147.

16. Maruyama S, Yoshioka H. Fujita K, Takimoto M, Satake Y. Sensitivity of group A streptococci to antibiotics. Prevalence of resistance to erythromycin in Japan. Am $J$ Dis Child 1979; 133: 1143-1145.

17. Seppälä H. Nissinen A, Yu Q, Huovinen P. Three different phenotypes of erythromycin-resistant Streptococcus pyogenes in Finland. J Antimicrob Chemother 1993; 32 $885-891$

18. National Committee for Clinical Laboratory Standards. Performance standards for antimicrobial susceptibility testing fourth informational supplement M100-S4. NCCLS Villanova. PA. 1992.
Many microbiological laboratories do not determine the antibiotic susceptibilities of group A streptococci. However, we suggest that such a test is indicated when a macrolide is considered as an alternative to penicillin. The disk diffusion and E-test appear to be suitable.

The authors acknowledge the technical help of B. N. van der Lubbe and T. J. K. van der Reijden. We thank G. P. Bieger-Smith for correction of the English text. We are very grateful to W. H. F. Goessens, A. Fleer, F. W. Sebens and L. H. M. Verhoef for supplying the group A streptococci from their laboratories in Rotterdam, Utrecht, Deventer and Enschede, respectively, and H. Seppälä for the erythromycin-resistant strain. Finally we thank Abbott, Pfizer and Roussel Uclaf for providing the antibiotic preparations, including disks and E-test strips.

19. Foulds G, Johnson RB. Selection of dose regimens of azithromycin. J Antimicrob Chemother 1993; 31 Suppl E: 39-50.

20. Phillips I, Acar J, Baquero $F$, Bergan $T$, Forsgren A, Wiedemenn B. ESGAB breakpoint determination: clarithromycin. Eur J Clin Microbiol Infect Dis 1991; 10: 993-994.

21. Mouton RP, Van Klingeren B (eds). Standaardisatie van gevoeligheidsbepalingen. Rijksinstituut voor de Volksgezondheid en Milieuhygiene (RIVM), Bilthoven, The Netherlands. 1985.

22. De Neeling AJ, Hemmes JH, Van Klingeren B (eds). Resistentie tegen antibiotica bij routine-isolaten van bacterien in zeven streeklaboratoria. $\quad$ Rapportnummer 253601001. Rijksinstituut voor de Volksgezondheid en Milieuhygiene (RIVM), Bilthoven, The Netherlands. 1993.

23. Eady EA, Ross JI, Cove JH. Multiple mechanisms of erythromycin resistance. $J$ Antimicrob Chemother 1990; 26: $461-465$.

24. Fernandes PB, Baker WR, Freiberg LA, Hardy DJ, McDonald EJ. New macrolides active against Streptococcus pyogenes with inducible or constitutive type of macrolidelincosamide-streptogramin B resistance. Antimicrob Agents Chemother $1989 ; 33: 78-81$.

25. Leclercq R, Courvalin P. Bacterial resistance to macrolide, lincosamide, and streptogramin antibiotics by target modification. Antimicrob Agents Chemother 1991; 35: 1267-1272.

26. Leclercq $R$, Courvalin $P$. Intrinsic and unusual resistance to macrolide, lincosamide, and streptogramin antibiotics in bacteria. Antimicrob Agents Chemother 1991; 35: 1273-1276.

27. Miyamoto Y, Takizawa K, Matsushima A, Asai Y, Nakatsuka S. Stepwise acquisition of multiple drug resistance by betahemolytic streptococci and difference in resistance pattern by type. Antimicrob Agents Chemother 1978; 13: 399-404.

28. Betriu C, Sanchez A. Gomez M, Cruceyra A, Picazo JJ. Antibiotic susceptibility of group A streptococci: a 6-year follow-up study. Antimicrob Agents Chemother 1993; 37: 1717-1719.

29. Scott RJD, Naidoo J, Lightfoot NF, George RC. A community outbreak of group A beta haemolytic streptococci with transferable resistance to erythromycin. Epidemiol Infect 1989; 102: 85-91.

30. Stingemore N, Francis GRJ, Toohey M, McGechie DB. The emergence of erythromycin resistance in Streptococcus pyogenes in Fremantle, Western Australia. Med J Austr 1989; 150: $626-631$

31. Seppälä H, Nissinen A, Järvinen $H$ et al. Resistance to erythromycin in group A streptococci. $N$ Engl J Med 1992; 326: 292-297.

32. Fernandes PB, Garmaise D. Resistance to erythromycin. $J$ Antimicrob Chemother 1987; 20: 449-450.

33. Järvinen $\mathbf{H}$, Nissinen $\mathbf{A}$, Huovinen $\mathbf{P}$. Erythromycin resistance in group A streptococci. Lancet 1989; 1: 1022-1023.

34. Holmström L, Nyman B, Rosengren M, Wallander S, Ripa T Outbreaks of infections with erythromycin-resistant group A streptococci in child day care centres. Scand J Infect Dis 1990; 22: 179 185 .

35. Wittler RR, Yamada SM, Bass JW, Hamill R, Wiebe RA 
Ascher DP. Penicillin tolerance and erythromycin resistance of group $\mathrm{A} \beta$-hemolytic streptococci in Hawaii and the Philippines. Am J Dis Child 1990; 144: 587-589.

36. Kirst HA, Sides GD. New directions for macrolide antibiotics: pharmacokinetics and clinical efficacy. Antimicrob Agents Chemother 1989; 33: 1419-1422.

37. Schrock CG. Clarithromycin vs penicillin in the treatment of streptococcal pharyngitis. $J$ Fam Pract $1992 ; 35$ : 622-626.

38. Still JG, Hubbard WC, Poole JM, Sheaffer CI, Chartrand S, Jacobs R. Comparison of clarithromycin and penicillin VK suspensions in the treatment of children with streptococcal pharyngitis and review of currently available alternative antibiotic therapies. Pediatr Infect Dis J 1993; 12 Suppl 3: S134-141.

39. Hooton TM. A comparison of azithromycin and penicillin $\mathrm{V}$ for the treatment of streptococcal pharyngitis. Am J Med 1991; 91 Suppl 3A: 23S-26S.

40. Moellering RC. Introduction: revolutionary changes in the macrolide and azalide antibiotics. Am J Med 1991; 91 Suppl 3A: 1S-4S. 